\title{
IGR J18483-0311: a new intermediate supergiant fast X-ray transient ${ }^{\star}$
}

\author{
F. Rahoui ${ }^{1,2}$ and S. Chaty ${ }^{1}$ \\ 1 Laboratoire AIM, CEA/DSM - CNRS - Université Paris Diderot, Irfu/Service d'Astrophysique, Bât. 709, CEA/Saclay, \\ 91191 Gif-sur-Yvette, France \\ e-mail: [frahoui; chaty]@cea.fr \\ 2 European Southern Observatory, Alonso de Córdova 3107, Vitacura, Santiago de Chile \\ Received 28 July 2008 / Accepted 8 September 2008
}

ABSTRACT

\begin{abstract}
Context. IGR J18483-0311 is a high-mass X-ray binary recently discovered by INTEGRAL. Its periodic fast X-ray transient activity and its position in the Corbet diagram - although uncertain - led to the conclusion that the source was probably a Be/X-ray binary (BeXB), even if a supergiant fast X-ray transient (SFXT) nature could not be excluded.

Aims. We aimed at identifying the companion star of IGR J18483-0311 and discriminate between a BeXB and a SFXT nature for this source.

Methods. Optical and near-infrared photometry, as well as near-infrared spectroscopy of the companion star were performed to identify its spectral type. We also assembled and fitted its broad-band spectral energy distribution to derive its physical parameters.

Results. We show that the companion star of IGR J18483-0311 is an early-B supergiant, probably a B0.5a, and that its distance is about 3-4 kpc.

Conclusions. The early-B supergiant nature of its companion star, as well as its fast X-ray transient activity indicate that IGR J18483-0311 has an SFXT nature. Nevertheless, the long duration and the periodicity of its outbursts, as well as its high level of quiescence, are consistent with IGR J18483-0311 being an intermediate SFXT, in-between classical supergiant X-ray binaries (SGXBs) characterised by small and circular orbits, and classical SFXTs with large and eccentric orbits.
\end{abstract}

Key words. infrared: stars - X-rays: binaries - stars: individual: IGR J18483-0311 - binaries: general - supergiants stars: fundamental parameters

\section{Introduction}

High-mass X-ray binaries (HMXBs) are X-ray sources for which high-energy emission is produced by accretion onto a compact object of material originating in a massive companion star. Until recently, the majority of known HMXBs were Be/X-ray binaries (BeXBs), and just a few were supergiant X-ray binaries (SGXBs). The launch of the INTErnational Gamma-Ray Astrophysics Laboratory (INTEGRAL, Winkler et al. 2003) in October 2002 completely altered the situation, as many more HMXBs with supergiant companion stars were discovered during the monitoring of the Galactic centre and the Galactic plane using the onboard IBIS/ISGRI instruments (Ubertini et al. 2003; Lebrun et al. 2003). Most of these sources were reported by Bird et al. (2007) and Bodaghee et al. (2007), and these studies revealed two main features that were not present in previously known SGXBs: first, many exhibit considerable intrinsic absorption, far higher than the interstellar level; second, some of these new sources were observed to have a transitory nature and occasionally exhibit a fast X-ray transient activity of duration of a few hours. It then appears that the INTEGRAL supergiant HMXBs can be classified into two classes: one class of considerably obscured persistent sources (see e.g. Chaty \& Rahoui 2007) and another of transitory sources called supergiant fast X-ray transients (SFXTs, Negueruela et al. 2006).

* Based on observations carried out at the European Southern Observatory under the Target of Opportunity programme ID 078.D-0268.
IGR J18483-0311 was discovered during observations performed by INTEGRAL in 2003 April 23-28 (Chernyakova et al. 2003), and was found to have average fluxes of about $10 \mathrm{mCrab}$ and $5 \mathrm{mCrab}$ in the $15-40 \mathrm{keV}$ and $40-100 \mathrm{keV}$ bands, respectively. Molkov et al. (2004) also detected the source in 2003 March-May during a survey of the Sagittarius arm tangent with INTEGRAL, and measured average fluxes of about $4.3 \mathrm{mCrab}$ and $3.9 \mathrm{mCrab}$ in the $18-60 \mathrm{keV}$ and the $60-120 \mathrm{keV}$ bands, respectively. By analysing archival data from the Rossi $X$-ray Timing Explorer (RXTE) All-Sky Monitor (ASM), Levine $\&$ Corbet (2006) also reported a 18.55 days periodicity of the lightcurve of IGR J18483-0311, which probably corresponds to its orbital period.

With archival data from observations performed by INTEGRAL between 2003 May and 2006 April, Sguera et al. (2007) discovered five new outbursts from IGR J18483-0311, whose activity lasted no more than a few days and was characterised by rapid flares lasting a few hours. They fitted its 3-50 keV spectrum with an absorbed cut-off power law, and derived $N_{\mathrm{H}} \sim 9 \times 10^{22} \mathrm{~cm}^{-2}$ (well above the galactic column density along the line of sight of about $\left.1.6 \times 10^{22} \mathrm{~cm}^{-2}\right), \Gamma \sim 1.4$, and $E_{\mathrm{c}} \sim 22 \mathrm{keV}$. They also demonstrated that all the outbursts can be described well by absorbed bremsstrahlung, whose parameters are $N_{\mathrm{H}} \sim 7.5 \times 10^{22} \mathrm{~cm}^{-2}$ and $k T \sim 21.5 \mathrm{keV}$. They reported a periodicity in the long-term ISGRI light curve of about 18.52 days - confirming the result of Levine \& Corbet (2006) - and argued that it is most likely an orbital period. They also derived a periodicity of about $21.05 \mathrm{~s}$ in the JEM-X 
light curve of the first outburst, which is likely a neutron star pulse period. Finally, from archival Swift observations, the authors obtained an accurate position of IGR J18483-0311, which allowed them to pinpoint its USNO B1.0 0868-0478906 and 2MASS J18481720-0310168 counterparts. They found that the magnitudes were typical of an absorbed massive late O/early B star which, considering the position of the source in the Corbet diagram and the periodicity of its high-energy activity, led them to conclude that IGR J18483-0311 was probably a BeXB, without excluding the possibility of an SFXT nature.

Chaty et al. (2008) assembled the broad-band spectral energy distribution (SED) of the companion star of IGR J18483-0311 from $0.7 \mu \mathrm{m}$ to $8 \mu \mathrm{m}$, with near-infrared (NIR) photometric observations performed at the ESO/NTT using the SofI instrument, as well as archival optical data from the USNO-A.2 catalogue, and mid-infrared (MIR) data from the GLIMPSE survey. They fitted the SED with a combination of two absorbed black bodies and showed that the best-fit function was consistent with the companion star being a heavily absorbed B star, confirming the HMXB nature of IGR J18483-0311. Finally, Masetti et al. (2008) performed optical spectroscopy of the companion star of IGR J18483-0311, and concluded that it was an O/B giant star because of the large equivalent width of the $\mathrm{H}_{\alpha}$ emission line.

In this paper, we report optical and NIR observations of IGR J18483-0311 performed in service mode at the ESO/NTT by our Target of Opportunity program ID 078.D-0268 (P.I. Chaty). These observations were designed to constrain the nature of both its companion star and the binary system. In Sect. 2, we describe the optical/NIR photometric and spectroscopic observations, as well as the data reduction. In Sect. 3, we present the results and show the broad-band SED of the companion star of IGR J18483-0311. We finally discuss our results and conclusions in Sect. 4.

\section{Observations and data analysis}

\subsection{Optical and NIR photometry}

In 2007 March 24, we performed optical photometry of IGR J18483-0311 in $U, B, V, R$, and $I$ bands with the imager SUSI2 installed at ESO/NTT. We used the large field of view $\left(5.5 \times 5{ }^{\prime} .5\right)$, and the plate scale was $0{ }^{\prime} .16$ per pixel. The exposure time was $60 \mathrm{~s}$ in all filters for each exposure. We also observed four photometric standard stars from the optical standard catalogue of Landolt (1992): PG1657+056, PG1657+056A, PG1657+056B, and PG1657+056C.

NIR photometry was carried out in $J, H$, and $K$ s bands with the spectro-imager SofI at ESO/NTT. We used the large field of view $\left(4^{\prime} .92 \times 44^{\prime} .92\right)$, and the plate scale was $0{ }^{\prime} .288$ per pixel. In each filter, the observations were obtained by repeating a set of images with 9 different $30^{\prime \prime}$ offset positions to subtract the sky emission, and the exposure time was $1.2 \mathrm{~s}$ for each position. We also observed three photometric standard stars from the faint NIR standard star catalogue of Persson et al. (1998): sj9116, sj9150, and sj9155.

The photometric data were reduced using IRAF by performing flatfielding and bad pixel correction, as well as bias subtraction in the optical bands, and crosstalk correction and sky subtraction in the NIR. After the reduction, all the images were aligned and combined. We then carried out aperture photometry on the optical images. In the NIR, we used the software StarFinder, part of the Scisoft package from ESO, well-suited for point-source photometry in crowded fields. The instrumental magnitudes were then transformed into apparent magnitudes using the zero-point, the extinction coefficient, and the colorterm corrections computed by linear fits using the standard stars. The optical and NIR magnitudes are listed in Table 1. We note that our optical magnitudes are about 1 mag lower than those given in the USNO catalogue, this difference probably being due to the large uncertainty ( $\geq 1 \mathrm{mag}$ ) of the USNO magnitudes for very faint sources ( $\geq 17$ in the $B$ band).

\subsection{NIR spectroscopy}

During 2007 March 18, 19, we performed NIR low resolution $(R \sim 1000)$ spectroscopy with SofI between $0.9 \mu \mathrm{m}$ and $2.5 \mu \mathrm{m}$, using the blue and red grisms and a $1^{\prime \prime}$ slit. Twelve spectra were acquired for each grism, half of then on source, and the other half with an offset of $30^{\prime \prime}$, allowing for sky emission subtraction. The exposure time of each spectrum was set to be $60 \mathrm{~s}$. In the same conditions, we also observed HIP 96265, a G3V telluric standard star, to be able to complete a telluric features correction.

The NIR spectra were reduced with IRAF by performing crosstalk correction, flatfielding, sky subtraction, and bad pixel correction. The target spectra were then extracted, wavelength calibrated by extraction of a xenon arc with the same setup, and finally combined. The telluric features were corrected by dividing the final target spectrum by the telluric standard. To avoid line contamination by the standard star, we finally multiplied the corrected spectrum by a solar spectrum provided by the National Solar Observatory (NSO), and modified using an IRAF routine developed by Maiolino et al. (1996). The routine corrects the solar spectrum for airmass, heliocentric, and rotational velocity to match the standard star properties, and smoothes the spectrum in such way that is is rebinned to the instrument resolution. The final spectrum of the companion star of IGR J18483-0311 - obtained by combining the blue and red grisms - is displayed in Fig. 1.

\section{Results}

\subsection{Spectral classification}

To identify the spectral type of the companion star of IGR J18483-0311, we compared our spectrum with spectra from the $H$ and $K$ band hot star classifications provided by Hanson et al. $(1996,2005)$ and the $K$ band spectroscopic classification described by Clark \& Steele (2000). In the spectrum displayed in Fig. 1, we indicate all detected features (listed in Table 2), most of them due to H I and He I. In the $H$ band spectrum, we also detected the $\mathrm{Pa} \beta(5-3)$ feature at $1.2822 \mu \mathrm{m}$ and the $\mathrm{O}$ I feature at $1.3170 \mu \mathrm{m}$.

The presence of the $\operatorname{Br} \gamma(7-4)$ line in absorption at $2.1668 \mu \mathrm{m}$ and several He I lines is typical of a normal 09-B3 massive star (Clark \& Steele 2000). This therefore excludes the possibility of the system being a BeXB. The presence of both the $\mathrm{He}$ I line at $2.0580 \mu \mathrm{m}$ in emission and the He I line at $2.1121 \mu \mathrm{m}$ in absorption is typical of early B0-B2 supergiants. Comparing the relative strengths of the He I lines at $1.6996 \mu \mathrm{m}$, 2.0580 and $2.1121 \mu \mathrm{m}$, and of the HI lines at $1.6819 \mu \mathrm{m}$, $1.7362 \mu \mathrm{m}$, and $2.1668 \mu \mathrm{m}$, with the relative strengths of the same lines in Hanson et al. (1996, 2005), we find that the star is probably a B0.5a supergiant.

\subsection{Spectral energy distribution}

To compile the broad-band SED of IGR J18483-0311, we added its archival MIR fluxes from the Spitzer's Galactic 
Table 1. Optical and NIR magnitudes (Mag) of IGR J18483-0311 obtained with SUSI2 and SofI. We also indicate the zero point $Z_{\mathrm{p}}$ in each filter.

\begin{tabular}{c|cccccccc}
\hline \hline Filters & $U$ & $B$ & $V$ & $R$ & $I$ & $J$ & $H$ & $K \mathrm{~s}$ \\
\hline$Z_{\mathrm{p}}$ & $23.279 \pm 0.023$ & $24.837 \pm 0.030$ & $24.423 \pm 0.018$ & $24.480 \pm 0.013$ & $22.707 \pm 0.061$ & $23.059 \pm 0.020$ & $22.814 \pm 0.013$ & $22.211 \pm 0.019$ \\
Mag & $>23.702$ & $>25.162$ & $21.884 \pm 0.313$ & $17.888 \pm 0.041$ & $14.382 \pm 0.061$ & $10.840 \pm 0.028$ & $9.376 \pm 0.018$ & $8.472 \pm 0.023$ \\
\hline
\end{tabular}

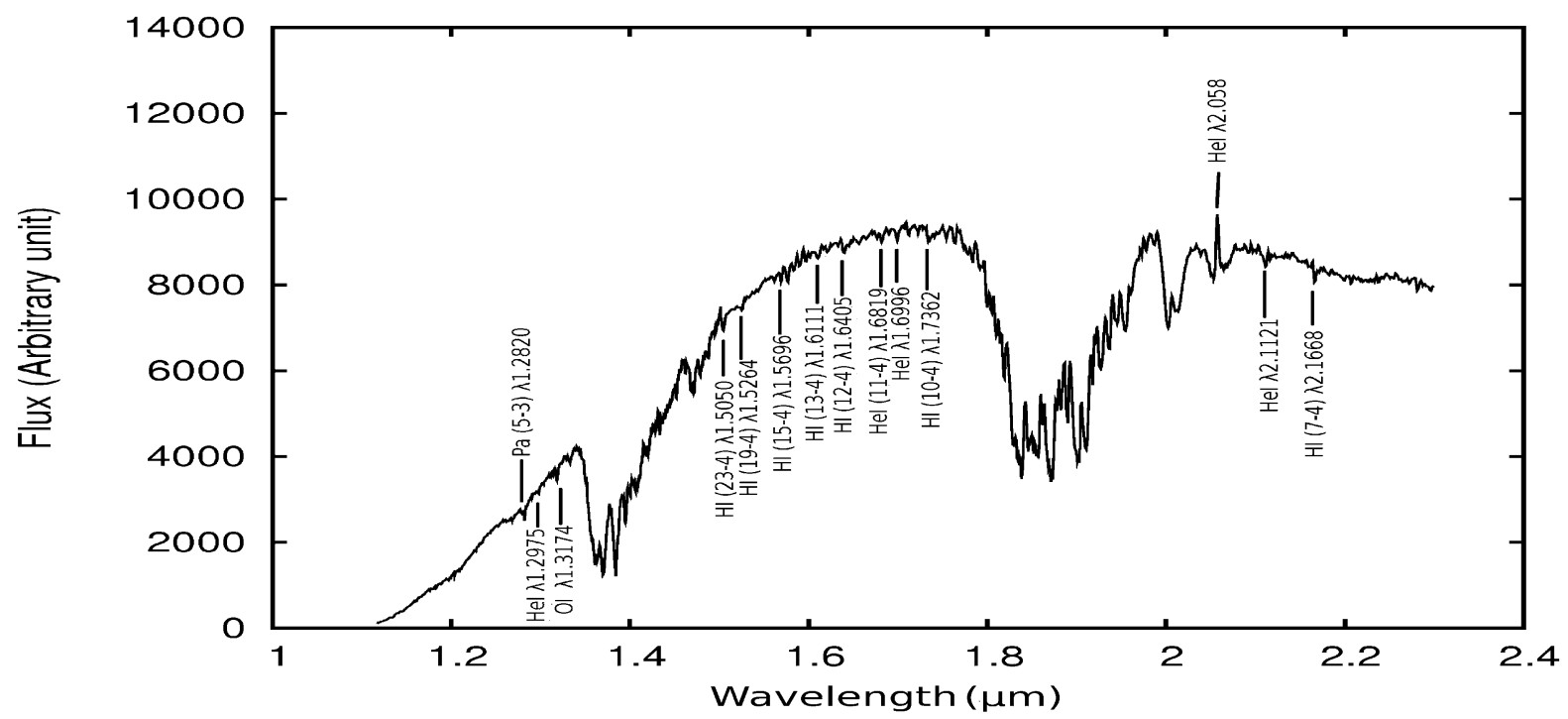

Fig. 1. $1.12 \mu \mathrm{m}$ to $2.3 \mu \mathrm{m}$ low resolution spectrum of the companion star of IGR 18483-0311, obtained using the blue and red grisms of SofI.

Table 2. Spectroscopic features we detected in our blue and red spectra of the companion star of IGR J18483-0311. We also provide their laboratory and fitted wavelengths in $\mu \mathrm{m}$ ( $\lambda$ and $\lambda_{\text {fit }}$, respectively), their equivalent width $(\stackrel{\circ}{W})$, and their full-width at half-maximum $(F W H M)$, in $\AA$.

\begin{tabular}{ccccc}
\hline \hline Identification & $\lambda$ & $\lambda_{\text {fit }}$ & $\stackrel{\circ}{W}$ & $F W H M$ \\
\hline $\mathrm{Pa}(5-3)$ & 1.2822 & 1.2820 & 1.013 & 8.351 \\
$\mathrm{He} \mathrm{I}$ & 1.2976 & 1.2975 & 0.765 & 20.97 \\
$\mathrm{O}$ I & 1.3165 & 1.3170 & 1.700 & 17.4 \\
$\mathrm{Br}(23-4)$ & 1.5043 & 1.5050 & 0.949 & 22.53 \\
$\mathrm{Br}(19-4)$ & 1.5265 & 1.5264 & 1.018 & 41.25 \\
$\mathrm{Br}(15-4)$ & 1.5705 & 1.5698 & 0.856 & 23.40 \\
$\mathrm{Br}(13-4)$ & 1.6114 & 1.6111 & 1.049 & 37.56 \\
$\mathrm{Br}(12-4)$ & 1.6412 & 1.6405 & 0.641 & 25.94 \\
$\mathrm{Br}(11-4)$ & 1.6811 & 1.6819 & 0.723 & 27.25 \\
$\mathrm{He} \mathrm{I}$ & 1.7007 & 1.6996 & 1.053 & 32.72 \\
$\mathrm{Br}(10-4)$ & 1.7367 & 1.7362 & 0.813 & 33.26 \\
$\mathrm{He} \mathrm{I}$ & 2.0587 & 2.0580 & -5.794 & 32.16 \\
$\mathrm{He} \mathrm{I}$ & 2.1126 & 2.1121 & 1.168 & 30.67 \\
$\mathrm{Br}(7-4)$ & 2.1661 & 2.1668 & 1.151 & 23.17 \\
\hline
\end{tabular}

Legacy Infrared Mid-Plane Survey Extraordinaire (GLIMPSE, Benjamin et al. 2003) of the Galactic plane $\left(|b| \leq 1^{\circ}\right.$ and between $l=10^{\circ}$ and $l=65^{\circ}$, on both sides of the Galactic centre) performed with the Spitzer Space Telescope with the IRAC camera in four bands, $3.60 \pm 0.38 \mu \mathrm{m}, 4.50 \pm 0.51 \mu \mathrm{m}, 5.80 \pm 0.73 \mu \mathrm{m}$, and $8.00 \pm 1.43 \mu \mathrm{m}$. The GLIMPSE fluxes of the companion star of IGR J18483-0311 are listed in Chaty et al. (2008). We then fitted the SED (using a $\chi^{2}$ minimisation) with an absorbed black body representing the companion star emission, following Rahoui et al. (2008). We added to the flux uncertainties a $5 \%$ systematic error in each NIR band, and a $2 \%$ systematic error

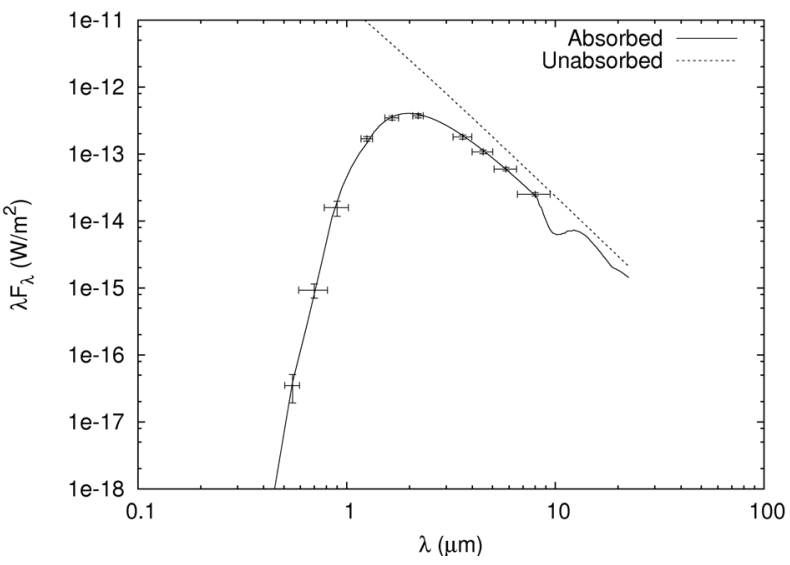

Fig. 2. Broad-band SED of IGR J18483-0311. Assembled with optical, near-, and mid-infrared data from SUSI2, SofI, and IRAC, respectively.

in each IRAC band, as given in the $\operatorname{SofI}^{1}$ and the IRAC ${ }^{2}$ manuals. The best-fit SED function and its parameters are displayed in Fig. 2 and listed in Table 3, respectively.

\subsection{Distance}

The best-fit temperature of the companion star of IGR 18483$0311\left(T_{*}=24600 \mathrm{~K}\right)$ is consistent with the star being a B0.5a supergiant, following the temperature calibration given by Searle et al. (2008). In their paper the expected unabsorbed absolute magnitude of a B0.5a supergiant star was indicated to be $M_{\mathrm{v}}=-6.5$, and its expected radius, $R_{\mathrm{e}}=33.8 R_{\odot}$. Using both of

\footnotetext{
1 http://WWW.1s.eso.org/docs/LSO-MAN-ESO-40100-0004/ LSO-MAN-ESO-40100-0004.pdf

${ }^{2}$ http://ssc.spitzer. caltech.edu/documents/som/som8.0. irac.pdf
} 
Table 3. Best-fit parameters of the SED of IGR J18483-0311. We indicate the total galactic absorption in the line of sight due to $\mathrm{HI}\left(A_{\mathrm{HI}}\right)$ and $\mathrm{H}_{2}\left(A_{\mathrm{H}_{2}}\right)$, the absorption of the source in the X-ray domain $A_{\mathrm{x}}$, and then the parameters: the optical intrinsic absorption $A_{\mathrm{v}}$, the temperature $T_{*}$ and the $R_{*} / D_{*}$ radius-to-distance ratio of the star. The absorptions are given in magnitudes.

\begin{tabular}{cccccc}
\hline \hline$A_{\mathrm{H}_{\mathrm{I}}}$ & $A_{\mathrm{H}_{2}}$ & $A_{\mathrm{x}}$ & $A_{\mathrm{V}}$ & $T_{*}\left[\Delta T_{*}\right](\mathrm{K})$ & $R_{*} / D_{*}$ \\
\hline 7.9 & 9.6 & 48.1 & $15.7_{-0.3}^{+0.2}$ & $24600[17500-36800]$ & $1.94_{-0.41}^{+0.39} \times 10^{-10}$ \\
\hline
\end{tabular}

these parameters, and $A_{\mathrm{v}}$ and $R_{*} / D_{*}$ we derived from our fitting, we can assess the distance of the source with one of the following relations: $D_{*}=10^{0.2\left(m_{\mathrm{v}}-A_{\mathrm{v}}-M_{\mathrm{v}}+5\right)}$ (in pc), or $D_{*}=\frac{R_{\mathrm{e}}}{R_{*} / D_{*}}$ (in $R_{\odot}$ ), where $m_{\mathrm{v}}$ is the optical apparent magnitude of the source. Both relations provide $D_{*}=3.44 \mathrm{kpc}$ and $D_{*}=3.93 \mathrm{kpc}$, respectively. Both values are consistent within the uncertainties and we therefore conclude that the distance of IGR J18483-0311 is about 3-4 kpc.

\section{Discussion and conclusions}

The identification of its companion star as a B0.5a supergiant, as well as its fast X-ray transient activity, are consistent with IGR J18483-0311 being an SFXT. Nevertheless, as already pointed out by Sguera et al. (2007), the X-ray behaviour of IGR J18483-0311 appears unusual. Whereas outbursts have a duration of a few hours and $L_{\max } / L_{\min } \sim 10^{4}$ for typical SFXTs, the outbursts of IGR J18483-0311 last a few days and its $L_{\max } / L_{\min }$ ratio is $\sim 10^{3}$ (implying that its quiescence is higher). Levine \& Corbet (2006) and Sguera et al. (2007) also reported a 18.52 days orbital period, which is low compared with expectations for classical SFXTs, of large and eccentric orbits.

Several authors emphasized the importance of clumpy winds in explaining the SFXT behaviour (in't Zand 2005; Leyder et al. 2007; Walter \& Zurita Heras 2007; Negueruela et al. 2008). They argued that the flares are created by the interaction of the compact object with dense clumps (created in the stellar wind of the companion star), the frequency of the flares depending mainly on the geometry of the system. In contrast, the quiescent emission is produced by the accretion onto the compact object of diluted inter-clumps medium, which would explain the low quiescence exhibited by classical SFXTs. By classifying twelve SFXTs in terms of the duration and the frequency of their outbursts, as well as their $L_{\max } / L_{\min }$ ratio, Walter \& Zurita Heras (2007) demonstrated the existence of intermediate SFXTs, characterised by a lower ratio and longer flares. Negueruela et al. (2008) proposed a general scheme to explain the emission of both SGXBs and SFXTs. The authors argued for the existence of a zone around the companion supergiant star (radius $\leq 2 R_{*}$ ), in which the clump density is relatively high, and another one (radius $\geq 3 R_{*}$ ) in which it is low. In terms of the orbital parameters of the compact object, the system could be a classical SGXB (small and circular orbit inside the zone of high clump density), a classical SFXT (large and eccentric orbit), or an intermediate SFXT (small orbits, circular or eccentric) with possible periodic outbursts (see also Sidoli et al. 2007, for an alternative model).
Considering the longer duration of its flares as well as their 18.52 days periodicity, and its lower $L_{\max } / L_{\min }$ ratio, IGR J18483-0311 is likely to be an intermediate SFXT. To be able to determine in the framework of the model proposed by Negueruela et al. (2008) whether its orbit is circular or elliptic, we can first consider the Kepler's third law for a B0.5a supergiant mass and radius $M_{*}=33 M_{\odot}$ and $R_{*}=33.8 R_{\odot}$ (Searle et al. 2008), and a neutron star mass of $1.4 M_{\odot}$, and derive the semi-major axis which we find to be $a \sim 2.83 R_{*}$. By assuming that IGR J18483-0311 only becomes active when orbiting within the zone of high clump density, we find that an eccentricity $0.43 \leq e \leq 0.68$ is needed to explain the average three-day bursting-activity reported by Sguera et al. (2007). We then conclude that IGR J18483-0311 is an intermediate SFXT with a small and eccentric orbit.

Acknowledgements. We are grateful to Jérôme Rodriguez for his useful website in which all INTEGRAL sources are referenced (http: //isdc . unige.ch/ rodrigue/html/igrsources.html). F.R. acknowledges the CNRS/INSU for the funding of the third year of his ESO/CEA PhD studentship. This research has made use of NASA's Astrophysics Data System, of the SIMBAD and VizieR databases operated at CDS, Strasbourg, France, of products from the US Naval Observatory catalogues, of products from the Two Micron All Sky Survey as well as products from the Galactic Legacy Infrared Mid-Plane Survey Extraordinaire, which is a Spitzer Space Telescope Legacy Science Program.

\section{References}

Benjamin, R. A., Churchwell, E., Babler, B. L., et al. 2003, PASP, 115, 953 Bird, A. J., Malizia, A., Bazzano, A., et al. 2007, ApJS, 170, 175 Bodaghee, A., Courvoisier, T. J.-L., Rodriguez, J., et al. 2007, A\&A, 467, 585 Chaty, S., \& Rahoui, F. 2007, Proceeding of the VIth INTEGRAL Workshop, The Obscured Universe, Space Research Institute, Moscow, Russia, 2006, ESA SP, 622

Chaty, S., Rahoui, F., Foellmi, C., et al. 2008, A\&A, 484, 783

Chernyakova, M., Lutovinov, A., Capitanio, F., Lund, N., \& Gehrels, N. 2003, The Astronomer's Telegram, 157, 1

Clark, J. S., \& Steele, I. A. 2000, A\&AS, 141, 65

Hanson, M. M., Conti, P. S., \& Rieke, M. J. 1996, ApJS, 107, 281

Hanson, M. M., Kudritzki, R.-P., Kenworthy, M. A., Puls, J., \& Tokunaga, A. T. 2005, ApJS, 161, 154

in't Zand, J. J. M. 2005, A\&A, 441, L1

Landolt, A. U. 1992, AJ, 104, 340

Lebrun, F., Leray, J. P., Lavocat, P., et al. 2003, A\&A, 411, L141

Levine, A. M., \& Corbet, R. 2006, The Astronomer's Telegram, 940, 1

Leyder, J.-C., Walter, R., Lazos, M., Masetti, N., \& Produit, N. 2007, A\&A, 465, L35

Maiolino, R., Rieke, G. H., \& Rieke, M. J. 1996, AJ, 111, 537

Masetti, N., Mason, E., Morelli, L., et al. 2008, A\&A, 482, 113

Molkov, S. V., Cherepashchuk, A. M., Lutovinov, A. A., et al. 2004, Astron. Lett., 30, 534

Negueruela, I., Smith, D. M., Reig, P., Chaty, S., \& Torrejón, J. M. 2006, in The X-ray Universe 2005, ed. A. Wilson, ESA SP, 604, 165

Negueruela, I., Torrejón, J. M., Reig, P., Ribó, M., \& Smith, D. M. 2008, in Am. Inst. Phys. Conf. Ser., 1010, 252

Persson, S. E., Murphy, D. C., Krzeminski, W., Roth, M., \& Rieke, M. J. 1998, AJ, 116, 2475

Rahoui, F., Chaty, S., Lagage, P.-O., \& Pantin, E. 2008, A\&A, 484, 801

Searle, S. C., Prinja, R. K., Massa, D., \& Ryans, R. 2008, A\&A, 481, 777

Sguera, V., Hill, A. B., Bird, A. J., et al. 2007, A\&A, 467, 249

Sidoli, L., Romano, P., Mereghetti, S., et al. 2007, A\&A, 476, 1307

Ubertini, P., Lebrun, F., Di Cocco, G., et al. 2003, A\&A, 411, L131

Walter, R., \& Zurita Heras, J. 2007, A\&A, 476, 335

Winkler, C., Courvoisier, T. J.-L., Di Cocco, G., et al. 2003, A\&A, 411, L1 